\title{
Philosophiques
}

\section{Du sens des choses à l'être des sens}

\section{Théodore Geraets}

Volume 41, numéro 2, automne 2014

URI : https://id.erudit.org/iderudit/1027228ar

DOI : https://doi.org/10.7202/1027228ar

Aller au sommaire du numéro

Éditeur(s)

Société de philosophie du Québec

ISSN

0316-2923 (imprimé)

1492-1391 (numérique)

Découvrir la revue

Citer ce document

Geraets, T. (2014). Du sens des choses à l'être des sens. Philosophiques, 41(2), 387-393. https://doi.org/10.7202/1027228ar d'utilisation que vous pouvez consulter en ligne.

https://apropos.erudit.org/fr/usagers/politique-dutilisation/ 


\section{Du sens des choses à l'être des sens}

\section{THÉODORE GERAETS}

Professeur émérite

Université d'Ottawa

Pour Jean Grondin, "la métaphysique est l'effort vigilant de la pensée humaine de comprendre l'ensemble de la réalité et ses raisons ${ }^{1} »$. Je trouve que Jean Grondin est trop généreux et que sa définition de la métaphysique lui permet de qualifier de métaphysique «toute grande philosophie qui a quelque chose de fondamental à dire sur ce qui est ${ }^{2} »$. Cela a comme avantage évident de pouvoir maintenir que la métaphysique n'est pas morte, même chez ceux qui se présentent comme des critiques sévères de la métaphysique.

À la dernière page de son livre, Jean Grondin nous place devant une alternative: entre une conception nominaliste selon laquelle le sens est « une production de la pensée qui projette ses concepts généraux sur le monde, lequel se compose de masses physiques individuelles, intrinsèquement dépourvues de sens » et celle "suggérée» par Gadamer, que «le sens que nous articulons en langage n'est pas une pure création de notre esprit, mais bien le sens des choses mêmes". Ce qu'il nous est permis de repenser ainsi: "c'est l'idée qu'il y a un sens au monde lui-même ${ }^{3}$ ".

Plus loin, je montrerai qu'il y a peut-être une troisième voie, proche de Gadamer, mais distincte de Grondin. Ici, je souligne seulement que, selon Jean Grondin, même le nominalisme «incarne une métaphysique ${ }^{4}$ ». Est-ce que sa façon de définir la métaphysique ne se fait pas au prix d'une sérieuse dilution $d u$ sens de ce terme?

Il est clair qu'une pensée comme celle de Kant est encore métaphysique au sens fort: des principes a priori de notre raison pratique permettent de conclure à l'existence de Dieu et à l'immortalité de l'âme ${ }^{5}$. Ce sens de transcendance qualifie certainement une pensée comme métaphysique au sens fort. Mais il y a lieu de penser qu'il peut y avoir des métaphysiques au sens fort, sans cette affirmation de l'existence de Dieu et de l'immortalité de l'âme.

Jean Grondin a aussi raison d'appeler la pensée de Heidegger une métamétaphysique, métaphysique en acte, dont les thèmes essentiels sont l'être et le divin ${ }^{6}$. "Pour ses premiers élèves, dont Löwith et Gadamer,

1. Du sens des choses, p. $2 \mathrm{I}$.

2. À l'écoute du sens. Entretiens avec Marc-Antoine Vallée, Bellarmin, 20 I I, p. 48.

3. Du sens des choses, p. I70.

4. Ibid., p. I3 I.

5. Cf. o.c., p. 9-I I, 49-50.

6. Cf. o.c., p. I3-20. 
Heidegger restera toute sa vie à la recherche de Dieu ${ }^{7}$. Et il eut des obsèques catholiques.

Un cas particulièrement intéressant est celui de Hegel. Comment situer Hegel ? A-t-il toujours continué à penser en métaphysicien ? Dans une contribution (provocatrice) à une Arbeitstagung en décembre $2007^{8}$, Walter Jaeschke a montré comment Hegel a abandonné la métaphysique.

Selon ce Mitarbeiter de I974 à I989, et directeur depuis I998 aux Archives Hegel, le grand philosophe qu'il a étudié toute sa vie aurait, après ses écrits de Jena, remplacé la metaphysica generalis par sa nouvelle logique objective ${ }^{9}$. Celle-ci n'est plus une théorie rationnelle de l'étant, mais une connaissance de soi de la raison, une théorie rationnelle du penser. «Préserver le concept de l'être pour quelque chose qu'on soupçonne au-delà de la conscience n'a plus de sens.» Pour cette pensée transcendantale radicale, " toute vérité n'est accessible que dans la conscience", "tout nous est accessible dans l'unité du penser ${ }^{10}$.

Bien sûr, cela ne veut pas dire que la conscience, la raison, ou l'esprit n'existent pas. L'esprit que nous sommes tous (der Geist der wir doch alle sind) cherche à se connaître en s'objectivant (de façon ultime, dans l'art, la religion et la philosophie $)^{11}$. Cela fait partie de la Realphilosophie qui remplace la metaphysica specialis.

Dans la discussion qui a suivi sa présentation, Jaeschke a dit: «Puisque nous avons dans le savoir des objets, nous avons le droit de parler de leur être. Mais pas dans le sens [...] que nous aurions là effectivement un être transcendant par rapport à la conscience, mais nous avons seulement un être qui est dans la conscience. L'objet n'est toujours qu'un objet connu et je n'ai jamais autre chose qu'un objet connu ${ }^{12}$.»

Quand Annette Sell, qui travaillait sous Jaeschke aux Archives Hegel, demanda s'il n'y aurait pas un moment métaphysique dans la Logique de Hegel, Jaeschke répondit que le prochain volume des cours de Hegel, un cours officiellement annoncé à l'université de Berlin comme étant Logik und Metaphysik, serait publié sous le nom de Logik. Et il refusa d'en discuter davantage: "Ich habe das schon für uns alle beschlossen! ${ }^{13}$ "

À la fin des années de Jena, Hegel a conçu sa science fondamentale comme une logique et non plus comme une métaphysique. Ajouter le mot métaphysique, dit Jaeschke, aurait pu induire en erreur et faire comprendre cette logique comme une ontologie. Sa logique couvre bien le même terrain, mais d'une toute nouvelle façon.

7. H.-G. Gadamer, Une biographie, Grasset, Paris, p. I I7-I I 8.

8. Wer denkt metaphysisch? dans Hegel's Aktualität, Joh. Kreuzer, W. Fink, 20 Iо.

9. O.c. p. I 59 .

10. O.c. p. I 62.

11. Cf. o.c. p. 164 .

12. O.c. p. I79.

13. O.c. p. I 80 . 
Il est vrai que quand Hegel parle de la logique comme du « royaume de la pensée pure ", il ajoute: "Ce royaume est la vérité elle-même, telle qu'elle est sans voile en et pour soi; pour cette raison, on peut dire: ce contenu est la présentation de Dieu tel qu'il est dans son essence éternelle, avant la création de la nature et d'un esprit fini ${ }^{14}$." Selon Jaeschke, ce "on peut dire» signifie, ici comme ailleurs, une distanciation plutôt qu'un rapprochement. Le Dieu de Hegel est tout à fait différent de celui de la métaphysique antérieure $^{15}$. Son nouveau programme d'une philosophie de la religion est, pour Hegel, d'un ordre totalement différent de celui de la theologia naturalis ${ }^{16}$.

Dans l'esprit objectif et absolu, nous n'avons toujours que le devenir d'une actualité créé par l'esprit (eine geistesgeschaffene Wirklichkeit). Ce que nous y rencontrons est esprit de notre esprit. Et, comme l'autre de l'esprit, la nature elle-même n'est qu'en tant que connue ${ }^{17}$.

Jaeschke résume ce fond transcendantal chez Hegel comme étant «ce savoir que tout ce à quoi nous avons affaire est savoir [dieses Wissen, daß

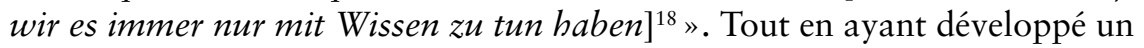
système englobant et complet, mais qui ne se prétendait pas exhaustif, Hegel n'aurait pas, dans ses années de pleine maturité, parlé en métaphysicien au sens fort: le transcendantal chez lui n'aurait pas ouvert une dimension de transcendance par rapport à la conscience.

Avant d'avoir pris connaissance de cette position récente, résolument anti-métaphysique et auto-critique, de Jaeschke, j'avais préparé en 20 I 2 un texte dont Jean Grondin fut le premier lecteur ${ }^{19}$. Dans ce texte, ma lecture de Hegel s'apparente à celle de Jaeschke, mais avec des différences fondamentales. Je résume ici une partie de ce texte, qui présente un «penser », différent de celui de Jaeschke, non soumis à la conscience.

Comme nous le savons, mais avons tendance à oublier, «penser» pour Hegel n'est pas qu'une simple activité humaine parmi d'autres; ce terme signifie plutôt ce qui rend humaine notre vie et toutes ses différentes formes: "Tout ce qui est humain est humain en ce que, et seulement en ce qu'il est produit par la pensée» (Enc. $\mathbb{S}$ 2). «Penser», en ce sens fondamental, se retrouve partout: voir, entendre, agir et réagir, se souvenir, intuitionner et même vouloir ou désirer, "tout cela, ce ne sont que des spécifications ultérieures de la pensée» (Enc. $\mathbb{} 24$ A; voir aussi TWA 7:46 s); "penser n'est pas quelque chose de particulier, une faculté spécifique, c'est plutôt le pouvoir essentiel et universel par lequel tout est produit» (VNM 6:278, I827-

14. Das Sein (I8I 2), Meiner, I986, p. I7.

15. Der Gott sieht nun wirklich anders aus, als der Gott der vorherigen Metaphysik, O.c., p.I $8 \mathrm{I}$.

16. Schlechthin inkommensurabel...O.c. p. I83.

17. Cf. o.c., p. I 87.

18. O.c. p. I 86.

19. «Hegel: The Articulation of Meaning - Reading Hegel after Gadamer », à paraître dans les Actes du XXIX ${ }^{\mathrm{e}}$ congrès du Internationale Hegel Gesellschaft à Istanbul. 
I 828 , cf. 3 I 3 ss, I 829-I $\left.830^{20}\right)$. Le penser est "partout et ce qui comprend tout [überall und das Allumfassende]»(VNM го: 3 ).

J'ai proposé d'exprimer et de comprendre «penser» en ce sens inclusif 21 comme «articulation de sens » ou, pour utiliser au-delà de leur contexte immédiat, les mots de Gadamer, comme "l'articulation intérieure de la Chose (qui est sens) » [die innere Gliederung der Sache (die Sinn ist)] (GW I : 477).

La philosophie est de toute évidence articulation de sens. Mais elle n'est pas simplement "penser » en son sens le plus inclusif. Hegel formule à la fois la différence et l'identité des deux:

En tant, cependant, que la philosophie est un mode particulier de penser, un mode par lequel celui-ci devient connaissance et connaissance qui comprend, la pensée propre à elle aura aussi un caractère différent de la pensée agissant dans tout ce qui est humain et même produisant l'humanité de ce qui est humain, tout autant qu'elle lui est identique, et qu'en soi il n'y a qu'une pensée $($ Enc $\mathbb{2}$ ).

Cette unité du penser, reconnue et comprise, toujours complète, mais jamais réalisée exhaustivement, est ce que Hegel appelle l'Idée. "L'Idée est le Vrai en et pour soi, l'unité absolue du concept et de l'objectivité. Son contenu idéel n'est aucun autre que le concept et ses déterminations; son contenu réel est seulement l'exposition de celui-ci qu'il se donne dans la forme d'un être-là extérieur... ». Voilà les sens et leur être. "L'être singulier est un côté quelconque de l'Idée, c'est pourquoi pour lui il est besoin encore d'autres effectivités; c'est seulement en elles toutes ensemble et dans leur relation que le concept est réalisé.» (Enc. $\mathbb{2} 2$ I3) L'articulation est réalisation et l'être est devenir. "L'Idée est essentiellement processus... » (Enc. $\int 2$ I 5 )

Quand j'entre par cette disputatio en dialogue avec Jean Grondin, je suis moi-même un sens, comme ce participant réel dans le processus d'articulation de sens. Jean lui-même ne saurait y échapper, et cela ne diminue en rien la réalité de l'un ou de l'autre. Voilà «l'esprit que nous sommes tous » (der Geist der wir doch alle sind).

L' univers herméneutique (WG 2: 44I, 466), dans son sens le plus inclusif, c'est-à-dire en tant qu'univers de sens, donc d'articulation de sens, en tant que compréhension, ainsi qu'en tant que réalisation de sens, celui-là même auquel Gadamer nous introduit, est comme une expression contemporaine de "l'Idée » à laquelle Hegel nous avait déjà introduits.

L'univers herméneutique est le monde, le tout de ce qui est (de ce qui, pour Hegel, devient), mais comme " un tout de sens ", qui est essentiellement

20. VNM (suivi du volume et de la page) renvoie aux Vorlesungen, Ausgewählte Nachschriften und Manuskripte, Meiner.

21. Cet usage est présent chez Hegel dans l'introduction des Leçons sur l'histoire de la philosophie de I 825-1826 et de façon plus développée dans les Leçons subséquentes de I 827 I 828 et I 829-I 830 , et aussi dans les éditions de I 827 et I 830 de l'Encyclopédie. 
«un infini de sens à développer et à interpréter» (eine Unendlichkeit des auszufaltenden und auszulegenden Sinnes) (GW I : 462).

Il est vrai que Gadamer se réfère ensuite à la métaphysique classique en évoquant "la relation transcendantale entre être et vérité, relation qui exige de penser la connaissance comme un moment de l'être même et non, originairement, comme conduite du sujet » (l.c.). Pourtant, l'univers de sens n'est pas pour lui « un ordre prédonné de l'être [eine vorgegebene Seinsordnung] dont les vrais rapports seraient étalés devant les yeux d'un esprit infini (de l'esprit créateur)» ( $G W$ I : 46I).

Tout ce qui est fait partie de l'univers de sens: une particule subatomique qui vient d'être découverte, une nouvelle galaxie, la possibilité réelle de nouvelles découvertes, tomber amoureux, mais aussi toute pensée en termes de "différence ", de "coupure» ou de "rupture».

Dans le Précis, Jean Grondin écrit: "La métaphysique est moins une théorie sur le monde que sur le sens du monde, c'est-à-dire sur ce qui rend la vie digne d'être vécue et vécue avec philosophie, c'est-à-dire avec une perspective métaphysique sur le sens des $\operatorname{choses}^{22}$.» Pour lui, cette perspective est que nous devons le sens inhérent au monde et à notre vie au Dieu créateur.

Ce Dieu créateur est absent dans la philosophie de Gadamer. L'auteur de la merveilleuse biographie de Gadamer a décrit avec une grande finesse l'attitude de son maître envers la religion. Gadamer lui a dit: "Je n'ai pas du tout la foi. Je le dis toujours avec un certain regret $^{23}$. » Avec Platon, il parle du "divin", ce qui exprime "surtout une conscience de la finitude, où la "transcendance" marquait avant tout la limite de ce que nous pouvons savoir ${ }^{24}$ ».

La question est de savoir si pour Gadamer l'être est transcendant. Il dit bien: "Ce petit espace de clarté [Dies bißchen Helligkeit] que nous parcourons dans notre conscience n'est pas la totalité de notre être ${ }^{25}$.» Est-ce que cet être, transcendant par rapport à la conscience, serait transcendant par rapport à l'univers de sens, d'articulation de sens, en tant que compréhension, ainsi qu'en tant que réalisation de sens? Je ne le crois pas.

Pour Jean Grondin, l'homme "veut comprendre quelque chose au sens des choses et de sa propre existence ${ }^{26} »$. Même quand il affirme qu'il y a un sens inhérent aux choses, ce sens apparaît « secondaire ». Ce qu'il y a d'abord, ce sont ces choses, ma propre existence, le monde. Je suis bien d'accord

22. Précis, p. 3.

23. Biographie, p. 30.

24. O.c., p. 28

25. Biographie, p. 27.

26. Du sens des choses, p. 8. 
quand il dit: "Nous avons accès aux choses, et celui qui le nie vit dans un univers qui n'est pas le mien ${ }^{27}$.»

Demandons alors: Qu'entendons-nous par «les choses », «ma propre existence", "le monde" ? Jaeschke dit que tout cela ne peut être que des objets connus. Mais je dirais plutôt d'eux qu'au lieu qu'ils aient un sens, ce sont des sens. Même mon genou, affecté par un cas léger d'osteo-arthrose, est un sens, «lourd " si l'on veut, mais un sens, faisant partie de l'univers de sens, mais défectueux dans son articulation intérieure.

Les «choses» sont des sens, mais toujours un sens qui est parce qu'il est articulé. C'est encore Hegel qui nous fait comprendre au tout début de sa Logique qu'un sens non articulé ( «l'être pur») n'est rien, ce qui résulte dans le devenir en tant que premier sens articulé (le premier concept), le vrai commencement de la logique.

Il s'agit donc de reconnaître l'être des sens dans leur articulation. Cette articulation de sens, ce "penser », et non «la conscience ", est le vrai "sujet» transcendantal.

Participer au devenir, qui est un processus d'articulation de sens, se fait nécessairement dans des conditions particulières, à tel point que Jean Grondin peut dire: "Ma foi n'est pas vraiment la mienne, elle est celle de la communauté de croyants à laquelle j'appartiens [...] C'est une foi reçue, transmise, acceptée, vérifiée, etc. ${ }^{28}$ » Un peu plus loin, Jean Grondin semble indiquer qu'il faut choisir entre la foi en Dieu et la foi au hasard ${ }^{29}$. Mais plus tôt il avait dit: "N'y a-t-il pas une "foi" en la raison dans l'exercice même de la rationalité ?»

La foi est toujours une relation à laquelle je participe sans pouvoir posséder (en pensée de survol) ni moi-même, ni cette relation, ni le terme avec lequel j’y suis relié. Cela est vrai de ce «premier principe» que beaucoup de philosophes ont tenu responsable de l'intelligence des choses, principe lui-même intelligent qui donne sens aux choses, comme étant "irriguées par un certain Sens ${ }^{30}$ ». Dans l'Entretien, Jean Grondin dit de la cinquième voie de saint Thomas, fondée sur le gouvernement des choses, qu'elle « reste très digne d'être méditée»(p. I 50). Mais faut-il remplacer la conscience constituante ou l'ego transcendantal par ce qui se trouverait de l'autre côté de la relation, que ce soit le Dieu créateur et/ou le souverain Bien? Manifestement, Jean Grondin le croit.

J'aurais bien aimé élargir mon propos en montrant la profonde parenté entre Hegel, lu par moi après Gadamer, et Merleau-Ponty pour qui le vrai

27. À l'écoute du sens, p. 43.

28. Ibid., p.I 53 .

29. Cf. o.c., p. I 53-I 54, et p. I33.

30. Cf. Du sens des choses, p.I33-I 34 . 
transcendantal est la vie ou l'expérience ${ }^{31}$. Pour lui, c'est la "foi perceptive" qui nous permet de voir chaque chose au-delà des sensations et chaque personne au-delà de ses qualités ${ }^{32}$. Et il y a pour lui l' «impossibilité du non-sens ou du vide ontologique ${ }^{33}$ ".

Dans une très belle page, Jean Grondin nous amène à sentir le sens dans la nature en parlant de la migration des bernaches et des saumons, articulateurs de sens. "Le sens directionnel, le sens signifiant et le sens sensitif existent donc déjà partout dans la nature et nous y avons part ${ }^{34}$.»

L'ontologie du sens vers laquelle Merleau-Ponty s'acheminait ne pouvait être qu'une endo-ontologie. C'est aussi une ontologie charnelle. Est-ce que "la chair» serait encore une autre expression de l'univers de sens de Gadamer et de l'Idée de Hegel ? Appeler leur pensée « un monisme qui se différencie " peut n'être qu'une «béquille " ${ }^{35}$, mais il y a une affinité profonde, trop peu reconnue, entre ces trois penseurs. Est-ce que la reconnaissance de cette affinité nous permettrait de dépasser les fausses alternatives et, libérés ainsi, de mieux faire notre part dans le processus de communication libre que Hegel appelle «l'esprit absolu»?

À la fin de l'entretien, Marc-Antoine Vallée demande: "Pourrait-on dire que l'expérience de la foi est celle d'un sens qui nous précède et qui nous fonde?". Jean répond: "Je ne pourrais mieux exprimer les choses... Mais si quelqu'un a une meilleure façon de penser les choses, je l'écouterai ou le lirai avec attention ${ }^{36}$."

Ce que j'ai tenté d'apporter ici est une autre façon de penser les choses; nous verrons si elle est jugée meilleure ou non. Pour moi, c'est la relationalité elle-même qui est le "premier principe » : l'univers de sens, cette articulation de sens, toujours déjà réellement à l'œuvre, comprenant le réseau englobant d'articulateurs de sens, humains ou autres (qui sont toujours euxmêmes des sens). C'est cela ma «foi philosophique».

31. Voir ma thèse, Vers une nouvelle philosophie transcendantale, Nijhoff, I97 I, p. I 8 I, I 86 .

32. Cf. "Le primat de la perception et ses conséquences philosophiques ", Bulletin de la Société française de philosophie, I947, p. II9-I 53.

33. Le visible et l'invisible, Gallimard, I964, p. I 56.

34. Du sens des choses, p. I40. Cela me fait penser à David Abram, éco-philosophe, qui s'est beaucoup inspiré de Merleau-Ponty.

35. Wer denkt metaphysisch? p. I 84 .

36. À l'écoute du sens, p. I 57. 